Methods We used the Hospital Episode Statistics - data covering all NHS-funded inpatient admissions, outpatient appointments and A\&E attendances in English hospitals. Children aged 10-17 first seen by a CP service between 2007/8 and 2016/17 were identified using service specialty codes that identify CP services. The presence of comorbid chronic conditions (e.g., cancer, neurological disorders or mental health conditions) in the five years prior to the first attended CP appointment (CP1) were identified from inpatient data using a validated ICD-10 code list. Service utilisation was described by histograms and rates of outpatient attendances, planned and emergency admissions and A\&E attendances. Analyses were conducted in R.

Results There were 3,185 (28\%) boys and 8,330 (72\%) girls. In boys, $24 \%$ lived in the most deprived fifth of neighbourhoods in the country and $17 \%$ in the least deprived fifth; in girls, no such gradient was observed with $20 \%$ in all fifths. Comorbidity prevalence was high: $55 \%$ of boys and girls had some other chronic condition in the five years prior to CP1. There was a rise in outpatient attendances, emergency admissions and A\&E attendances in the two years prior to CP1, with rises in emergency admissions and A\&E attendances most striking. For example, the rate of emergency admissions 21-24 months prior to CP1 was 11.7 (95\% CI 10.8, 12.6) per 100 person-years; in the three months prior to CP1, it was 38.3 (95\% CI 36.7, 39.9). All abated after CP1. There was a spike of planned admissions after CP1 with a flat trend beforehand.

Conclusion Children presenting to CP services exhibit high degrees of physical and mental comorbidity. We found patterns of service utilisation indicative of unmet need prior to CP1 that warrants further investigation. Though our results may not generalise to all children with $\mathrm{CP}$, this study suggests that earlier identification and referral could reduce unnecessary A\&E visits and emergency admissions.

\section{Mental Health}

\section{RF07 RELEASE OF 13 REASONS WHY AND HOSPITAL- PRESENTING SELF-HARM IN IRELAND}

1,2P Corcoran*, 'E Griffin, ${ }^{1,2} \mathrm{CB}$ Dillon, ${ }^{1} \mathrm{~N}$ McTernan, ${ }^{2} \mathrm{AP}$ Fitzgerald, ${ }^{1,2} \mathrm{E}$ Arensman. ${ }^{1}$ National Suicide Research Foundation, National Suicide Research Foundation, Cork, Ireland; ${ }^{2}$ School of Public Health, University College Cork, Cork, Ireland

\subsection{6/jech-2019-SSMabstracts. 122}

Background Portrayals of suicidal behaviour in the media have been linked with copycat suicidal behaviour, a phenomenon referred to as the Werther Effect. In recent years, media reporting of high-profile suicides and portrayals of fictional suicides in film/television have been associated with increased deaths.

The Netflix series 13 Reasons Why was released on 31 March 2017 and received a lot of public attention, particularly for its detailed portrayal of the suicide of the female protagonist. We examined whether there was any evidence of an increase in hospital-presenting self-harm in Ireland following the release of the series.

Methods The National Self-Harm Registry Ireland records selfharm presentations to all Irish hospital emergency departments. Case ascertainment and data collection are carried out by trained data registration officers who follow standard operating procedures. We examined the temporal pattern of hospital-presenting self-harm by persons aged $\geq 10$ years, stratified by sex and whether the self-harm involved sharp objects as depicted in 13 Reasons Why or other means. Poisson regression was used to compare the incidence in the three months after the series' release compared to the rest of the calendar year 2017 and to make the same comparison for each of the preceding six years.

Results During 2011-2017, there was an annual average of 11,515 self-harm presentations to hospital from an annual average population of 3.99 million persons aged. Self-harm involving sharp objects accounted for one in four presentations $(2,937$ per year, 25.5\%) and had an age-standardised female and male incidence rate of 79 and 75 per 100,000 person-years, respectively. Three quarters of presentations involved other means (8,578 per year, $74.5 \%)$, primarily intentional drug overdose, with a female and male incidence rate of 245 and 197 per 100,000 person-years, respectively.

The incidence of female self-harm involving sharp objects was 100 per 100,000 in the three months after the release of 13 Reasons Why, 20\% higher than the incidence in the rest of 2017 (incidence rate ratio, $\mathrm{IRR}=1.20 ; 95 \%$ confidence interval $=1.08-1.33$ ). Such an increased rate was not observed for the same period in any of the previous six years (IRR range $=0.95-1.07)$. The same three-month period showed no notable change in incidence in any year for male self-harm involving sharp objects or for female or male self-harm involving other means.

Conclusion The study findings show evidence of an increase in female hospital-presenting self-harm involving sharp objects following the release of the 13 Reasons Why series.

\section{RF08 PERCEIVED SEX DISCRIMINATION, MENTAL HEALTH AND WELLBEING: A PROSPECTIVE ANALYSIS OF THE UK HOUSEHOLD LONGITUDINAL STUDY}

RA Hackett*, A Steptoe, SE Jackson. Behavioural Science and Health, University College London, London, UK

\subsection{6/jech-2019-SSMabstracts.123}

Background Perceived sex discrimination is linked with poor mental health. However, previous research is dominated by small convenience samples of US students, with a focus on sexual harassment rather than discrimination more broadly. This study aimed to examine cross-sectional and prospective associations between perceived sex discrimination and mental health and wellbeing in a community sample from the United Kingdom (UK).

Methods Data were from 2956 women aged $\geq 16$ years participating in the UK Household Longitudinal Study. Perceived discrimination was reported in 2009/10. Psychological distress, mental functioning, life satisfaction and self-rated health were assessed in 2009/10 and 2013/14. Depression was assessed in 2009/10. Linear and logistic regression analyses adjusted for age, household income, education and ethnicity.

Results Perceived sex discrimination was reported by 576 (19.5\%) participants. Cross-sectionally, perceived discrimination was associated with increased depression (Odds ratio $(\mathrm{OR}=3.16$, 95\% Confidence Interval (CI) $2.10 ; 4.79)$ and psychological distress $(B=1.26,95 \%$ CI $0.95 ; 1.56)$, and poorer mental functioning $(B=-5.39,95 \%$ CI -6.33 ; -4.46), life satisfaction $(B=-0.52,95 \%$ CI $-0.69 ;-0.36)$ and self-rated health 
$(\mathrm{OR}=1.89,95 \%$ CI $1.47 ; 2.41)$. Prospectively, perceived sex discrimination was associated with increased psychological distress $(B=0.66,95 \%$ CI $0.07 ; 1.24)$, poorer mental functioning $(B=-1.37,95 \%$ CI $-2.71 ;-0.03)$ and lower life satisfaction $(B=-0.32$, 95\% CI $-0.58 ;-0.05)$ over four-year follow-up.

Conclusion Women in the UK who perceive that they have been discriminated against on the basis of their sex report poorer mental health and wellbeing than those who do not perceive sex discrimination. These results provide cross-sectional and prospective evidence of associations between perceived sex discrimination and mental health and wellbeing outcomes in UK adults.

\section{RF09 SOCIO-ECONOMIC INEQUALITIES IN SUICIDE: CAUSATION OR CONFOUNDING? A MORTALITY FOLLOW-UP OF FORTY POPULATION CENSUSES FROM TWELVE EUROPEAN COUNTRIES}

V Lorant*. Institute of Health and Society, UCLouvain, Brussels, Belgium

10.1136/jech-2019-SSMabstracts.124

Background Socio-economic inequalities in suicide remain substantial and persistent in most European countries. The mechanism driving these inequalities, however, remains obscure. Two causal mechanisms have been attributed varying degrees of importance: low socio-economic status may directly increase the risk of suicide (hereafter 'causation') or low socio-economic status and suicide may share confounders (hereafter, 'confounding'). This paper aims to assess whether educational inequalities in suicide (EIS) are due to causation or to confounding.

Methods The DEMETRIQ study collected and harmonised register-based data on mortality follow-up of forty population censuses from fifteen Northern, Southern, Western, and Eastern European populations. More than 89,554 suicides were registered over 300 million person-years. Four tests of causation $v s$ confounding were implemented. Test 1: whether a decreasing (increasing) EIS over the lifecycle supports confounding (or causation). Test 2: whether greater (lower) EIS in males than in females supports confounding (or causation). Test 3: At the country level, whether EIS is more related to the Gini income inequality index (causation) or to the proportion of suicides that take place among the younger age group (confounding). Test 4 applied an instrumental variable approach that exploits changes in the legislation on compulsory educational age to instrument educational status. Tests 1 and 2 were performed with multilevel Poisson Regressions (SAS), test 3 with linear regression, and test 4 used two-stage Poisson Regression (STATA).

Results For test 1, we found that educational inequalities in suicide decreased over the life cycle. The risk of suicide among the less educated as compared to the more highly educated declined from $\mathrm{RR}=1.85$ (95\% CI: 1.6-2.07) in those aged $35-39$ to $\mathrm{RR}=1.27(95 \mathrm{CI} \%, 1.12-1.44)$ for those aged $75-79$. Test 2 indicated that educational inequalities in suicide were systematic and of greater magnitude in males (all countries, $\mathrm{RR}=2.51,95 \% \mathrm{CI}: 2.44-2.58$ ) than in females (all countries $\mathrm{RR}=1.32$, 95\%CI: 1.26-1.38). For test 3, EIS decreased with mean age of suicide (std Beta $=-0.59$, t-test $=-5.0$ ) and increased with the Gini coefficient (std beta $=0.47$, t-test $=2.8$ ). Test 4 indicated that there was no association between higher education and suicide.
Conclusion We found stronger support for the confounding explanation than for causation. Educational inequalities in suicide should be addressed by early targeting of vulnerable groups who struggle to complete their education.

\section{RF10 HIDDEN HARMS OF HYPNOTICS: A POPULATION BASED RECORD LINKAGE STUDY OF PSYCHOTROPIC MEDICATION AND SUICIDE RISK}

${ }^{1}$ IN Onyeka*, 'A Maguire, ${ }^{1,2} \mathrm{D}$ O'Reilly. ${ }^{1}$ Centre for Public Health, Queen's University Belfast, Belfast, UK; ${ }^{2}$ Administrative Data Research Centre Northern Ireland, Centre for Public Health, Queen's University Belfast, Belfast, UK

\subsection{6/jech-2019-SSMabstracts. 125}

Background Suicide is a leading cause of death worldwide with rates of suicide higher in Northern Ireland (NI) compared to the rest of the UK and Ireland. In addition, the consumption of psychotropic medication is increasing. The association between psychotropic medication and suicide risk is contested. Existing population-based studies that have examined whether types of psychotropic medications are associated with suicide are either restricted to older populations or have investigated each medication separately rather than comparing them directly to one another. This study examined the association between psychotropic medication types and death by suicide across the whole adult population.

Methods A cohort of 1,115,647 individuals aged 18-75 was identified from the 2011 Census records, linked to prescription records for the five months surrounding the census and followed until the end of 2015 to determine suicide risk. All individual, household and area-based attributes were derived from census records and selected on the basis of known suicide risk. Five categories of psychotropic medication were identified; none, antidepressants, anxiolytics, hypnotics and antipsychotics. Logistic regression was used to construct models to test the association between specific types of psychotropic medication (singularly and in combinations) and death by suicide adjusting for sociodemographic and physical health characteristics. Several sensitivity analyses were conducted taking into consideration the different timing, amount/threshold of medication and changes in medications consumed.

Results About $14 \%$ of the cohort $(n=153,319)$ had received $\geq 2$ prescriptions of any psychotropic medication in the five months around Census. Eight hundred and seventy persons ( $n=870)$ died by suicide during 2011 to 2015. Suicide was higher in those on psychotropic medication than in those without $(0.24 \%$ versus $0.05 \%$ respectively). In the fully adjusted logistic regression model, association between death by suicide and any psychotropic medication record remained statistically significant $(\mathrm{AOR}=3.69$, 95\%CI: 3.13-4.35). However, individuals solely on hypnotics had the highest AOR of those on singular medication $(\mathrm{AOR}=4.24,95 \% \mathrm{CI}$ : 2.96-6.06 compared to just $\mathrm{AOR}=2.22$, 95\%CI: $1.75-2.82$ for those on antidepressant medication only). In terms of medication combinations, use of both anxiolytics and hypnotics $(\mathrm{AOR}=8.76$, 95\%CI: 5.93-12.94) and a combination of antidepressants, anxiolytics and hypnotics $(\mathrm{AOR}=8.53,95 \% \mathrm{CI}$ : 5.62-12.94) were the strongest predictors of deaths by suicide.

Conclusion Risk of death by suicide varied by medication type with hypnotic medications making an important contribution to death by suicide. Although previous work has focused on antidepressant therapy and suicide risk, those on hypnotic medication are an at-risk group that warrant further attention. 